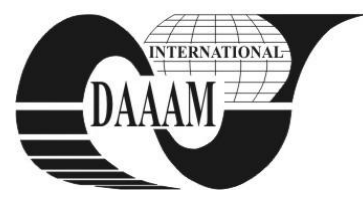

\title{
APPLYING VALUE STREAM MAPPING TO IDENTIFY HIDDEN RESERVES AND AVOID BOTTLENECKS
}

\author{
DURANIK, T[omas]; STOPPER, M[arkus] \& RUZBARSKY, J[uraj]
}

\begin{abstract}
In manufacturing and production companies the implementation of lean production processes is a major goal in order to increase profitability. In this paper three states of the manufacturing process are outlined, based on the method of Value Stream Mapping.

For the current state we will identify processes that produce any type of "waste" (activities that do not add any value). Then we will describe an ideal state showing only zero-waste processes and finally a future state that could give answers on how to improve the production flow and efficiency, to reduce production costs and to increase flexibility.
\end{abstract}

Key words: value stream mapping, effective lean management, manufacturing bottleneck, lead and cycle time reduction

\section{INTRODUCTION}

Nowadays every company puts a lot of emphasis on product quality. The secret of success is no longer only to meet required quality criteria, but also to satisfy customer needs by providing a large product range, short lead times and on time delivery. In order to achieve these goals it is necessary to optimize the manufacturing process by implementing a lean production process that intends to shorten the time between suppliers and customers by eliminating waste in the production chain using the Value Stream Mapping method.

\section{PROBLEM STATEMENT}

Assessing the actual situation, the company shows too many release failures and a waste production that is too high thereby exceeding delivery deadlines. As long as these problems are not solved, resources have to be increased in order to handle failure releases. Due to long delivery times the company might additionally face losses of revenue or business by missing critical customer deadlines or paying SLA penalties.

\section{GOAL AND APPLICATION AREA}

Application of Value Stream Mapping helps to ensure that all manufactured products reach the customers without any defects in compliance with delivery dates and without the production of any waste. This can be achieved by increasing efficiency, control and productivity in the production. (Ishiwata, J. 1991)

This case study shows the application of Value Stream Mapping on production processes in a medium sized manufacturing company.

\section{VALUE STREAM MAPPING (VSM)}

Value Stream Mapping is a tool commonly used in lean continuous improvement programs to help understand and improve the material and information flow within organizations. As with any lean management toolset the principle aim of Value Stream Mapping is to improve processes. (Murman E, et al 2002) This is achieved by highlighting areas of waste generation within a process, thereby enabling businesses to eliminate these activities. Value Stream Mapping also has the benefit of categorizing process activity into two main areas: value add, non value add (but necessary)/waste.

1) A value adding process is any step in the production process that improves the product for the customer.

2) Non-Value Add (NVA)/Waste. Non-value adding is an activity that consumes time and/or resources and does not add value to the service or product for the customer. These activities should either be eliminated, simplified, reduced or integrated.

Hines, P. and Rich, N. (1997) defined the following "7 Wastes": Overproduction, Delay, Transporting, OverProcessing, Inventory, Motion and Making Defective Parts.

\section{APPLYING VALUE STREAM MAPPING}

In any organization products will follow an exact stream or a similar path from beginning to end. In a first step all existing value streams have to be identified. Then a single specific value stream is chosen which is called the model line. It serves as a model for all the other value streams. (Arbulu, J. R. and Tommeilein, D. I., 2002). After choosing a representative the value stream map can be drawn. The various steps of value stream mapping are shown in Fig. 1, Fig. 2 and Fig. 3.

\subsection{Current state map}

Critical point at the beginning of each improvement is the clear specification of the product value as it is delivered to the end customer.

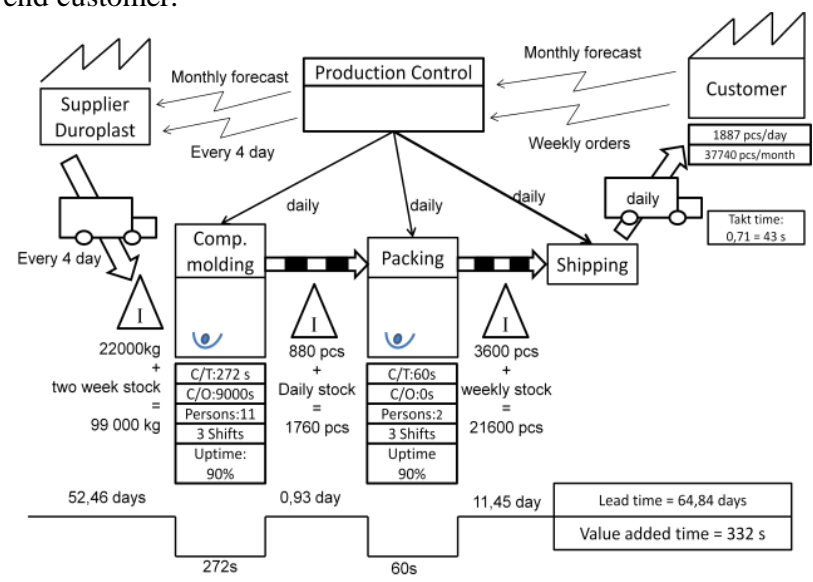

Fig. 1. Current state of VSM

Therefore in order to map the customer different questions need to be answered (What is the monthly requirement?, How many pieces are produced in one day? etc.). In this specific example daily demand of the customer is 1.887 pcs. Orders come in on a weekly basis and delivery to the customer is daily. Value adding comprises of two processes: compression molding and packaging. The lead time is 68,84 days and the 
value adding time 332 s. Out of these numbers we get a tact time of $43 \mathrm{~s}$. The ratio of continuous work time to value adding time indicated as the percentage of the total production time is $0,0055 \%$.

\subsection{Ideal state map}

The ideal state map means respectively describes processes with zero waste.

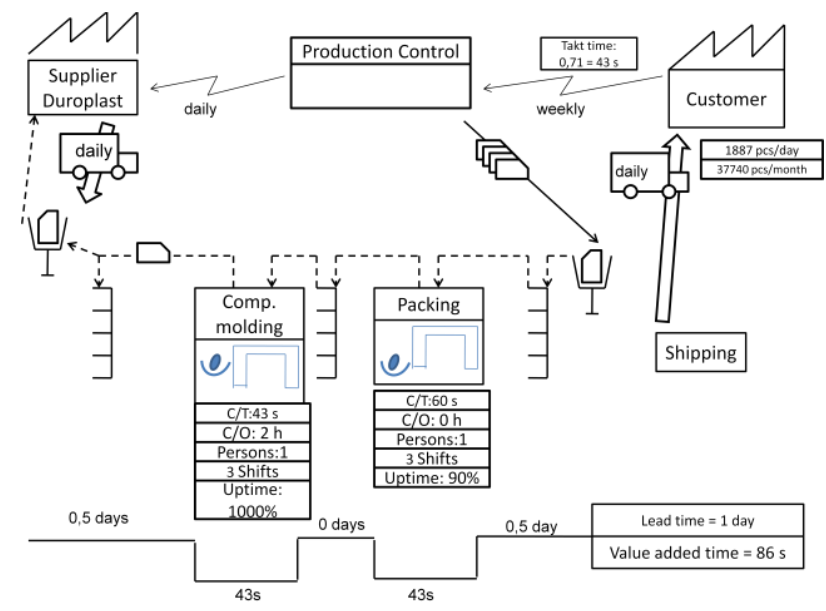

Fig. 2. Ideal state of VSM

In this context the word "ideal" means that in production reality it may not be really feasible to reach this optimum state in the near future. The ideal state does not take into account that the distance to the supplier might be far, that a daily supply with duroplast raw material cannot be granted as the material has to dry for two weeks in stock, that the production is also dependent on material quality and that the duration of compression molding itself cannot be influenced. In the ideal state of VSM the lead time is 1 day and the value adding time 86 seconds. The lead time has improved from 64,84 to 1 day and the ratio of continuous work time to value adding time indicated as the percentage of the total production time is $0,0994 \%$, as compared to a ratio of $0,0055 \%$ in the current state, which is an improvement of more than 18-times.

\subsection{Future state map}

In the future state map of VSM questions about supporting improvements, material flow, customer delivery strategy and information flow are answered. (Lu, David J. 1989)

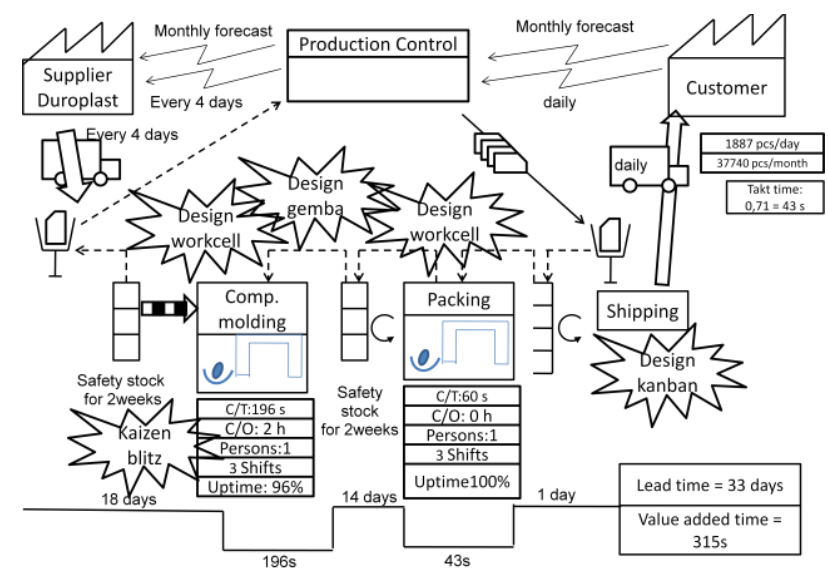

Fig. 3. Future state of VSM

After developing the current state into the future state lead time is reduced from 68,84 days to 19 days using the kanban method of lean management for the synchronization of production and material flow. The ratio of continuous work time to value adding time indicated as the percentage of the total production time is $0,00838 \%$, which is an improvement of more than 1,5-times over the present state.

\subsection{Identification of the bottleneck process}

The bottle neck process is the operation with the longest cycle time within the registered overall tact time. In this example the whole tact time is 43 seconds with the process of compression molding displaying the longest cycle time. The compression molding time cannot be reduced to 43 secound because the technology itself would not allow such a reduction. With a compression time of 43 seconds the quality of the product would get so low that it would not be sellable to the customer anymore.

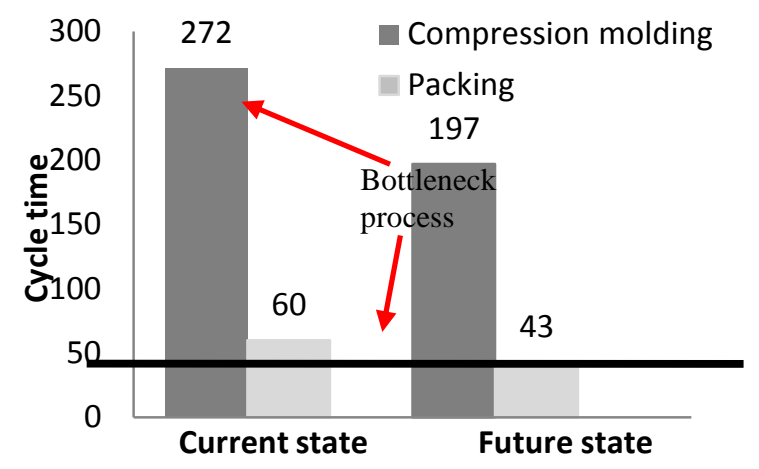

Fig. 4. Identifying the bottleneck process

\section{CONCLUSION}

In order to continuously improve lean production processes future state scenarios should outline the target that the current state scenario should strive to achieve. VSM has proven to be a highly useful tool that helps not only to eliminate specific waste generating processes within a production cycle but also identifying other waste generating processes for future elimination, thereby creating a habit or culture of lean production management. By using the VSM method we were able to achieve the following results:

- $\quad$ Lead time reduction of $70 \%$

- $\quad$ Productivity increase of $28 \%$

- Inventory decrease of about $70 \%$

These results can be further improved by the future introduction of integrated automation and control systems (Pasko, J. and Gaspar, Š. 2007). As the company strives to become a "lean manufacturer" in the future values streams will be analyzed by applying VSM thereby defining bottleneck processes and improving overall production efficiency.

\section{REFERENCES}

Arbulu, J. R. e Tommelein, D. I., Value Stream Analysis of Construction Supply Chains: Case Study on Pipe Supports Used in Power Plants.In: Annual conf. Int. Group for lean construction, 10th , 2002, Gramado, BR. Proceedings... Gramado, BR, Aug 06- 08, 2002

Hines, P. and Rich, N. (1997). "The seven value stream mapping tools", International Journal of Operations \& Production Management, Vol. 17 Iss: 1, pp.46 - 64

Ishiwata, J. (1991). Productivity through Process Analysis, Productivity Press, Cambridge, MA, 1991

Murman E, et al. Lean Enterprise Value, p 72. Palgrave, 2002

Lu, David J. (1989) Kanban: Just In Time At Toyota, Japan Management Association, Productivity Press, 1989

Pasko, J. and Gaspar, Š. 2007. Theory of the welding process automation. In: European and international standards in technical documentation. Prešov: ELBA, 2007 s. 148-151 